\title{
Novel Platform for Ocean Survey and Autonomous Sampling using Multi-agent System
}

\author{
Tawfiq Taher ${ }^{1}$, Gabriel D. Weymouth ${ }^{2}$, Tony Varghese ${ }^{3}$ \\ Center for Environmental Sensing and Modeling, \\ Singapore-MIT Alliance for Research and Technology Centre, \\ Singapore 138602. \\ Email: ${ }^{1}$ tawfiq@ smart.mit.edu, ${ }^{2}$ weymouth@ mit.edu, ${ }^{3}$ tony@ smart.mit.edu
}

\begin{abstract}
In-situ surveying and sampling of ocean environments provides critical data for laboratory work and oceanographic research. However, sampling a time-varying ocean field is often time and resource limited-meaning that samples often miss the features of interest. This paper presents a modular autonomous multi-agent robotic system which has been developed to accommodate a variety of research activities. This paper demonstrates the complementary capabilities of the agents by simultaneously surveying a time-varying coastal environment and using that information to obtain a sparse but representative set of water samples. This autonomous system enables the elimination of redundant resources, thereby reducing the overall cost of at-sea sampling and improving sample quality.
\end{abstract}

\section{INTRODUCTION}

Using in-situ instruments, fundamental physical oceanographic parameters such as conductivity, temperature, chlorophyll can be collected with a fairly easier logistics setup but it is more challenging to analyze biological parameters such as micro-organisms and nutrients in such manner. Some of the recent research work demonstrates the capability of very diverse in-situ analysis [1], [2] but most of them are not comprehensive or lack mobility. Collecting a physical sample is still a feasible option for those who do not have access to such advance tools or require parameters that are not measured by in-situ instruments. In the process of collecting water samples there is a chance of mixing or disturbing the water while accessing it during a manual / human intrusion. Discrete water sample collection using autonomous vehicles is not a new concept [3], [4] but usually involves complex mechanisms, comparatively expensive and is not necessary for all applications. Traditional water sample collection involves Niskin bottles [5] which is deployed and operated manually. There are devices with large number of arrays of Niskin bottle which are actuated electrically [6] but they require larger logistics support for deployment. We demonstrate an adaptive and inexpensive autonomous water sampling capability with decentralized multi-agent intelligent strategy.

\section{PLATFORM}

Center for Environmental Sensing and Modeling (CENSAM) has developed a mobile network of sensors using inexpensive autonomous surface vehicles (ASV) as our mulitagent platform [7]. These were developed by engineers at

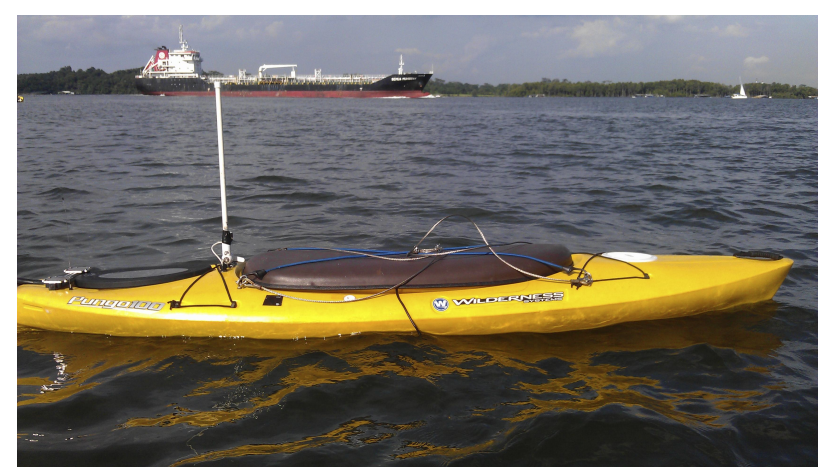

Fig. 1: Autonomous Surface Vehicle in the sea.

Massachusetts Institute of Technology's (MIT) Department of Ocean Engineering in order to facilitate surface and undersea oceanographic monitoring. The ASVs use kayaks equipped with computers, electronics and mechanical propulsion mechanisms.

\section{A. ASV Hardware}

The hull of an ASV is a $3 \mathrm{~m}$ long regular ocean kayak which is manufactured by Pungo (model Pungo100) as illustrated in the figure 1 . The main compartment has a low-density polyethylene lid cover that has a rubber seal to prevent most of the water from entering the kayak hull. A $245 \mathrm{~N}$ thruster produced by Minn Kota is used for the propulsion while a steering servo is used for steering of the ASV. The main vehicle computer is a fit-pc2 from Compulab and the payload computer is Hurricane-QM57 from LiPPERT Embedded Computers. An internal thermostat controlled fan is included for passive cooling. Communication and control is possible with a remote controlled radio and receiver pair. The Global Positioning Sytem (GPS), compass and Inertial Measuring Unit (IMU) are integrated in a sensor box that is mounted onto each kayak. The entire unit is powered by lithium iron phosphate (LiFePO4) Prismatic Cell from A123 systems which has the energy capacity of $160 \mathrm{AH}$ approximately. Multiple batteries can be connected in parallel configuration if higher capacity is required. 


\section{B. ASV Software}

The ASVs are operated with Mission Oriented Operating Suite (MOOS) [8] and MOOS Interval Programming (MOOSIvP) [9], [10] software packages as our middleware autonomy solution. MOOS is an operating suite which basically is a placeholder for the sensor database, it is also known as MOOSDB. MOOS is also responsible for the communication in between software packages. MOOS-IvP in an extension of MOOS that provides behavior based autonomous control. With MOOS-IvP, prior to deployment, the setup can be tested by simulating the actual field environment. Also it provides monitoring of the ASVs location, status, communication, power e.t.c., during field operation.

Our low-level drivers and algorithms are integrated with MOOS-IvP. MOOS convention was followed while naming the packages as the prefixes $i, p, u$ stands for instrument, program and utility respectively.

\section{Sensors}

Other than the essential navigational sensors such as compass, GPS and IMU. ASVs can be deployed with various kinds of payload sensors for specific experiment needs. In our experiment set up, we have used ASVs equipped with in-situ oceanographic environmental sensors and in-house developed automated water sample collector.

1) Oceanographic Environmental Sensors: We have used YSI Sonde 6600 V2 [11] for our oceanographic sensors as illustrated in the figure 2. The YSI Sonde could be configured to collect wide variety of ocean parameters. Our usual configuration of the YSI Sonde with the specifications are: Conductivity range: 0 to $100 \mathrm{mS} / \mathrm{cm}$ with accuracy of $\pm 0.5 \%$, Temperature range: -5 to $+45^{\circ} \mathrm{C}$ with accuracy of $\pm 0.15^{\circ} \mathrm{C}$ , Depth range: 0 to 656 feet (0 to 200m) with accuracy of \pm $0.3 \mathrm{~m}, \mathrm{pH}$ range: 0 to 14 unit with accuracy of \pm 0.2 unit , Dissolved Oxygen range: 0 to $50 \mathrm{mg} / \mathrm{L}$ with accuracy of \pm $2 \%$, Turbidity range: 0 to 1000 NTU with accuracy of $\pm 5 \%$, Chlorophyll range: 0 to $400 \mu \mathrm{g} / \mathrm{L}$.

We have developed a software package, iYSI, which communicates with the YSI Sonde and registers the data in the MOOS sensor database in real time.

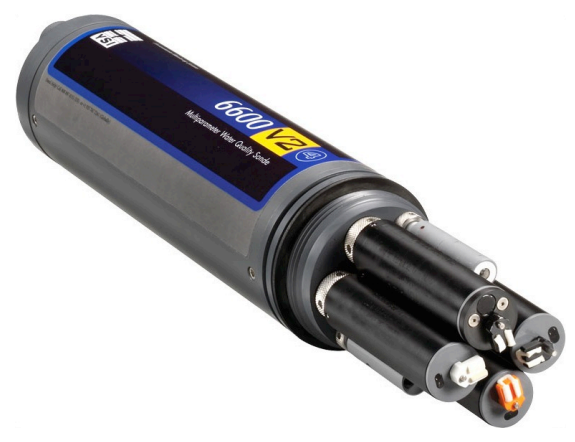

Fig. 2: YSI Sonde 6600 V2 [11]

2) Automated Water Sample Collector: Automated water sample collector is a small array of Niskin bottles, as illustrated in the figure 3 , operated by electronics circuitry. We have used Model 1010 Niskin Water Sampler from General Oceanics

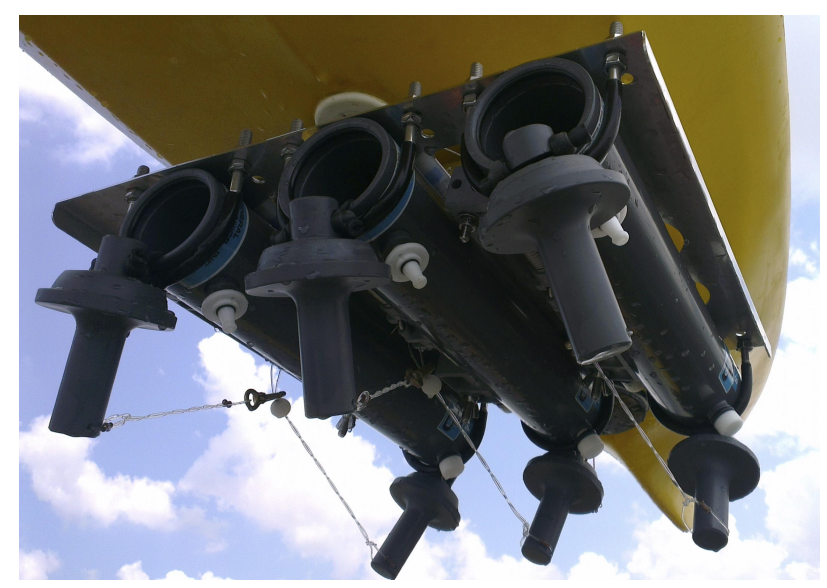

Fig. 3: Automated water sample collector.

as our sample collecting bottles. These bottles are actuated automatically with the help of servo motors (Dynamixel Rx-64 from ROBOTIS). We have developed a servo motor controller software iNiskin, which is discretely able to actuate the Niskin bottles mounted under the ASV. This software communicates with MOOS database consistently and waits for instruction from any other software component to actuate the bottles. This water sample collector does not have any depth control hence it only collects near surface water samples which was intended and suits the specific experiments it was designed for.

\section{EXPERIMENT}

The hardware and software described above was used to conduct a set of field tests for multi-agent sampling of an ocean system. CENSAM's marine research is focused in the coastal zone of Singapore. Specifically north east part of the coast, also known as the Johor Strait, is more diverse in terms of variety of physical and biological oceanographic features. Johor Strait is polluted from industrial effluents, human settlements and processing plants near the coast and the rivers [12]. However, there is a large establishment of mariculture industry in the

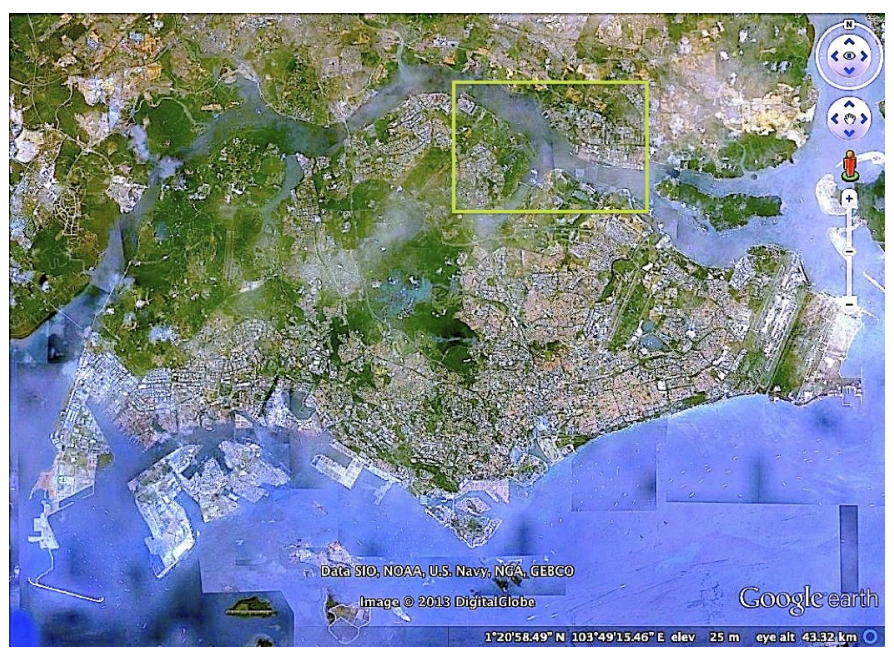

Fig. 4: Singapore. [15] 
Johor Strait [13]. Unfortunately, there are incidents of fish kills in the recent past and since, monitoring Harmful Algal Blooms (HAB) is part of our current research topics [14], this region is one of the most suitable area for our experiments. These trials were conducted in the coastal waters of Singapore, where the dependance of micro-biological growth on local temperature and nutrient conditions is of interest, and is illustrated by a green box, in the the figure 4 [15]. We have performed our experiments near to the river mouths where the chances of HAB occurrence is higher [14]. The basic concept of these tests was to use one ASV to take a sparse survey of the field (to increase the speed) while the second ASV used that data to determine where to take water samples.

In our experiment set up, we have used two ASVs. They were equipped with oceanographic environmental sensors (ASV A) and automated water sample collector (ASV B) as seen in figure 5(a) and 5(b) respectively.

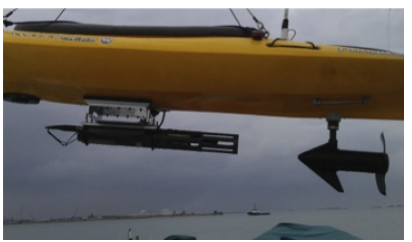

(a) Oceanographic sensors

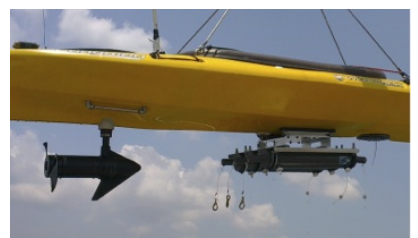

(b) Water sample collector.
Fig. 5: ASV equipped with sensors for deployment.

The state diagram in the figure 6 explains the high level work flow of the process. Both of the ASV A and B are deployed and while ASV A is used for in-situ measurements, Surveing in the ocean, ASV B is standing by at Parking state for collecting water samples as seen in the screenshot taken from the remote monitor and control interface of the vehicles in the figure 7(a). Once ASV A finishes its mission, collected data is analyzed on board with pre-specified preferences of parameters and goes to Parking state. Depending on the analyzed results, an adaptive decision is taken by ASV A for

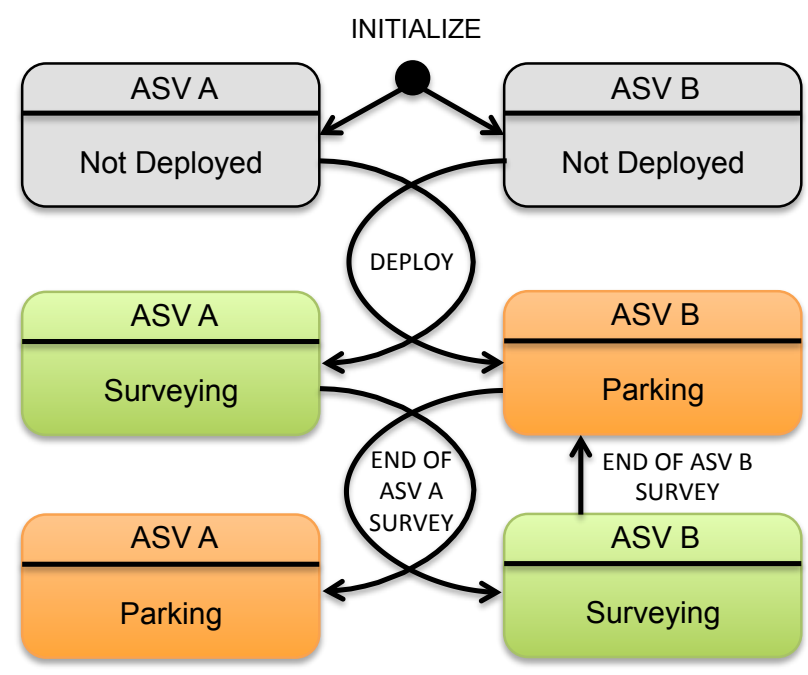

Fig. 6: State diagram of the process.

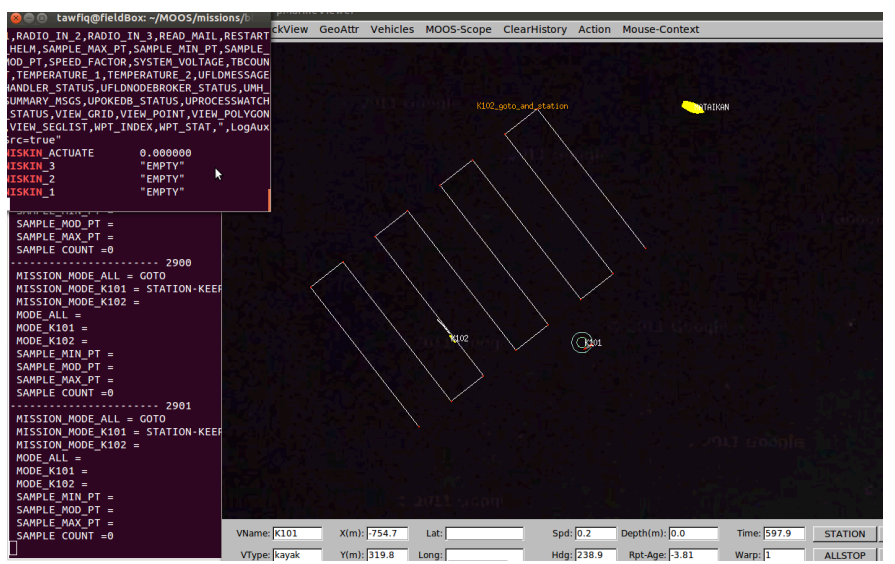

(a) ASV A in Surveing mode.

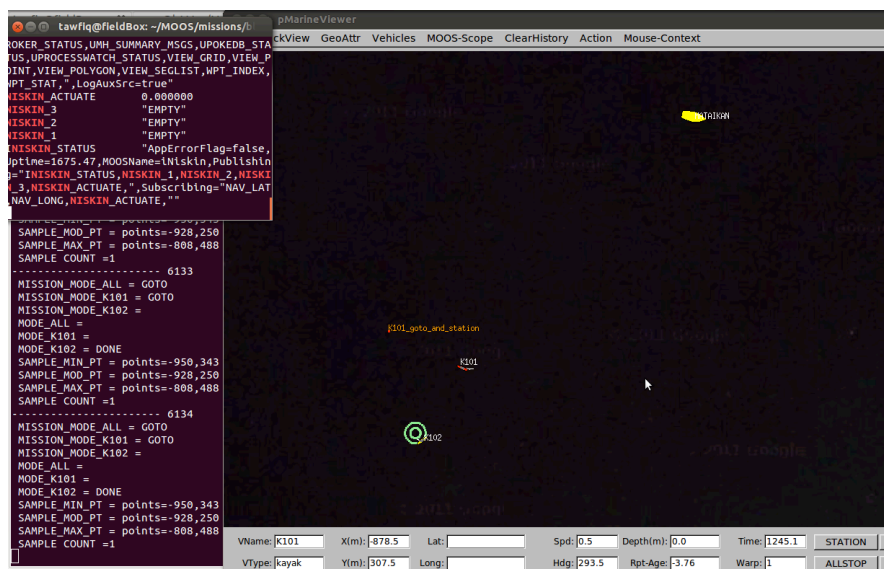

(b) ASV B in Surveing mode.

Fig. 7: Realtime screenshot of the ASVs during an experiment.

ASV B to collect water samples at three key locations and ASV B goes into Surveing mode as in the figure 7(b). ASV B then goes back to Parking state after all the samples have been collected.

A simplistic approach for deciding the points of water sample collection was chosen. Since we are limited by collecting three samples only, so we have identified three zones of interest containing three different measurement/concentration of the parameter being investigated, which are the Ambient, Minimum and Maximum. Ambient Zone is the area with most common measurement in a particular dataset. We observed the frequency distribution of the dataset and determined that our Ambient Zone sample should be taken from the modal class. Minimum Zone and Maximum Zone was identified as the class containing the smallest and largest measurements respectively.

\section{RESUlTS}

The operation of the ASVs were challenging due the high volume of marine traffic and leisure activities. They are to be monitored constantly with safety chase boats. Moreover, the sea current along the Johor Strait is very strong for ASV operation, so in difficult conditions it takes longer to finish its 


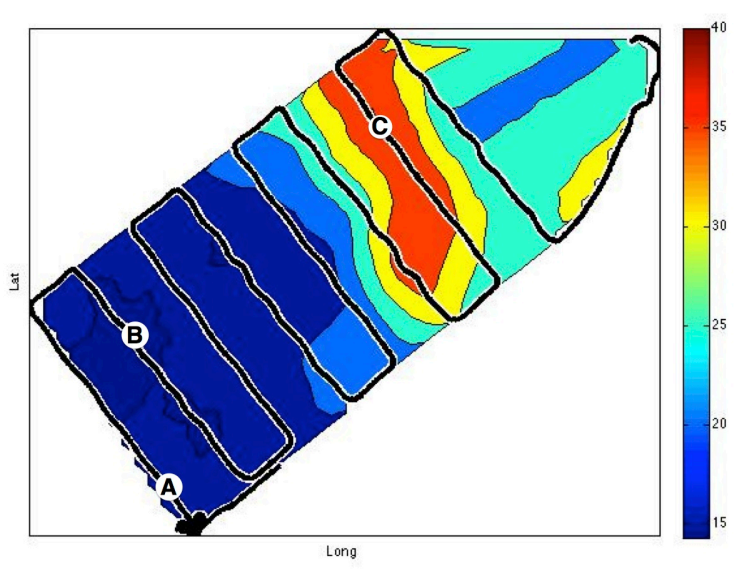

(a) Interpolated spatial data.

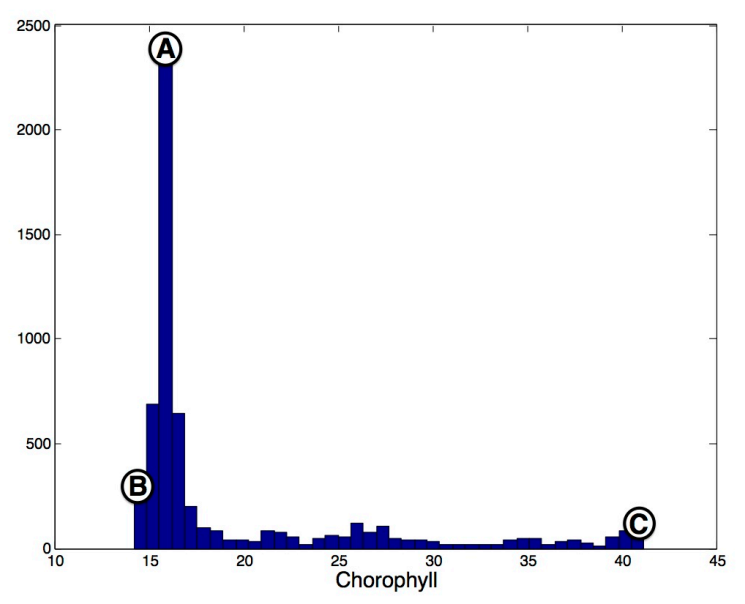

(b) Histogram of the data.

Fig. 8: Chlorophyll $(\mu \mathrm{g} / \mathrm{L})$ concentration from an experiment.

mission. In this section, the data obtained from the experiments are analysed and the approach of adaptive decision algorithm is validated against the field experiment data.

Spatial distributed data of a field experiment was acquired from the ASV A and plotted in the figure 8(a) with cubic interpolation. In this specific experiment Chlorophyll $(\mu \mathrm{g} / \mathrm{L})$ concentration was measured. As it can be observed from the color coded scale, red patch has the highest Chlorophyll concentration and can be identified as the Maximum zone. The Minimum zone also can be easily identified from the darkest blue patch. In fact, these two zones are easy to identify by only performing simple sorting on the data set. On the other hand, it is tricker to identify the actual Ambiemt zone in the provided boundary condition. If the histogram plot is observed in the figure 8 (b) of the same set of experimental data, the primary mode is distinctly identified from the skewed distribution. In such a case, Ambient zone sample should be from a location associated with most commonly available value of the scoped parameter, in this case Chlorophyll, which evidently lies in the modal class.

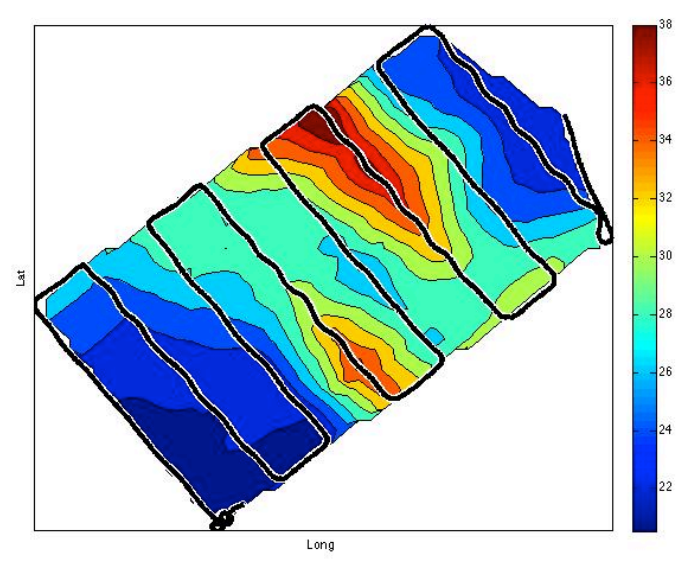

(a) Interpolated spatial data.

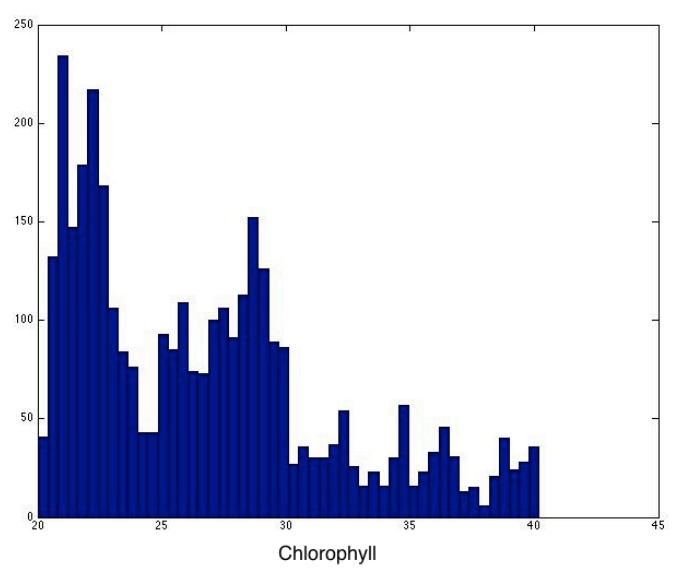

(b) Histogram of the data

Fig. 9: Chlorophyll ( $\mu \mathrm{g} / \mathrm{L})$ concentration from an experiment.

The three numbers of water samples collected by the ASV B are illustrated in the figure $8(a)$ and $8(b)$ as $A, B$ and $C$ representing for Ambient Zone, Minimum Zone and Maximum Zone respectively. When both the figures are compared, we can verify the validity of the water sample collection. Sample $A$ falls in the modal class with a measurement of Chlorophyll at $15.6(\mu \mathrm{g} / \mathrm{L})$ as seen in the histogram of the figure $8(\mathrm{~b})$. Hence sample $A$ was taken from the Ambiemt zone. The concentration of sample $B$ was $14.2(\mu \mathrm{g} / \mathrm{L})$ which was the lowest measured value of the data set and therefore is considered as the Minimum zone. Similarly, the concentration of sample $C$ was $41.2(\mu \mathrm{g} / \mathrm{L})$ which was the highest measured value of the data set and therefore is considered as the Maximum zone.

\section{CONCLUSION}

This work presents the use of a set autonomous ASV for intelligent sampling of a time-varying ocean field. A simple strategy was used to determine which locations should be sampled. This strategy gives three samples, minimum, maximum and ambient concentration of a parameter, which are commonly required by the ocean scientists. With this 
current system, the manual process of decision making and collecting samples are completely eliminated. Because of the simultaneous deployment of multiple ASVs and rapid deployment process, time difference between measured parameter and water sample collected is very minimal which results in acquisition of more accurate data set. Also, the whole process being automated, more numbers of experiment can be performed within the given time, eventually higher efficiency and lower cost in logistics expenses.

Although, the decision making approach works fairly well in most scenarios such as in figure 8(a), in a more complex scenario where multiple mode exists, further advanced algorithm is to be used to improve the data set accuracy. As we can see in the figure 9(a), there are multiple areas with high concentration of Chlorophyll which gives an indication of the Maximum zones but yet still it is hard to determine the Ambient Zone as it is illustrated in the the histogram from the figure 9(b). There are multiple number of modes, with little difference in number of occurrences of the parameter. Therefore, identifying one of them as the Ambient zone might be misleading. In such cases, more intelligent deceision making algorithm can be introduced to improve system performance.

Though we have implemented an algorithm which might not be efficient enough to handle all kind of scenarios, it still serves as a demonstration of the infrastructure which has been developed. This system is a platform for diverse type of sensors with resources to be distributed and shared among multiple mobile intelligent nodes which facilitates similar research activities. With further persistent development to the platform and decision making algorithms, the quality of data and samples provided will continue to grow, while maintaing the low operational costs.

\section{ACKNOWLEDGMENT}

The research described in this WAVES (Water and Air Vehicles for Environmental Sensing) Lab project was funded in whole by the Singapore National Research foundation (NRF) through the Center for Environmental Sensing and Modeling (CENSAM) at Singapore-MIT Alliance for Research and Technology (SMART) Centre.

\section{REFERENCES}

[1] Adornato, L., et al. "In situ nutrient sensors for ocean observing systems."Proceedings of the Oceanobs 09: Sustained Ocean observations and Information for Society Conference. Vol. 2. 2009.

[2] Scholin, C., et al. "The Environmental Sample Processor (ESP)-an autonomous robotic device for detecting microorganisms remotely using molecular probe technology." OCEANS 2006. IEEE, 2006.

[3] Bird, L. E., Alana Sherman, and John Ryan. "Development of an active, large volume, discrete seawater sampler for autonomous underwater vehicles." OCEANS 2007. IEEE, 2007.

[4] Dodd, P. A., et al. "Collection of water samples from an autonomous underwater vehicle for tracer analysis." Journal of Atmospheric and Oceanic Technology 23.12 (2006): 1759-1767.

[5] http://en.wikipedia.org/wiki/Nansen_bottle

[6] Grasshoff, K., Klaus K., and Manfred E., eds. Methods of seawater analysis. Wiley-Vch, (2009): 63

[7] Curcio, J., Leonard, J. and Patrikalakis, A., "SCOUT-a low cost autonomous surface platform for research in cooperative autonomy." OCEANS, 2005. Proceedings of MTS/ IEEE. IEEE, 2005.

[8] Newman, P.M. "MOOS-mission orientated operating suite." Massachusetts Institute of Technology, Tech. Rep 2299.08 (2008).
[9] Benjamin, M. R., et al. "Nested autonomy for unmanned marine vehicles with MOOSIvP." Journal of Field Robotics 27.6 (2010): 834-875.

[10] Balasuriya, A., et al. "Behavior-based planning and prosecution architecture for autonomous underwater vehicles in ocean observatories." OCEANS 2010 IEEE-Sydney. IEEE, 2010.

[11] http://www.ysi.com/

[12] Koh, H., Poh-Eng L., and Zamali M. "Management and control of pollution in Inner Johore Strait." Environmental Monitoring and Assessment 19.1-3 (1991): 349-359.

[13] Chou, R., and H. B. Lee. "Commercial marine fish farming in Singapore.” Aquaculture Research 28.10 (1997): 767-776.

[14] Leong, S. C. Y., Pavel T., and Nicholas M. P. "Monitoring harmful algal blooms in Singapore: Developing a HABs observing system." OCEANS, 2012-Yeosu. IEEE, 2012.

[15] https://maps.google.com/ 